\title{
Spin-flipping with an rf-dipole and a full Siberian snake*
}

\author{
A.M.T. Lin $^{1 * *}$, B.B. Blinov ${ }^{1}$, Ya.S. Derbenev ${ }^{1}$, T. Kageya ${ }^{1}$, \\ D.Yu. Kantsyrev ${ }^{1(a)}$, A.D. Krisch ${ }^{1}$, V.S. Morozov ${ }^{1(a)}$, J.R. Murray ${ }^{1}$, \\ D.W. Sivers ${ }^{1(b)}$, V.K. Wong ${ }^{1}$, K. Yonehara ${ }^{1}$, \\ V.A. Anferov ${ }^{2}$, C.M. $\mathrm{Chu}^{2}$, P. Schwandt ${ }^{2}$, B. von Przewoski ${ }^{2}$, \\ V.N. Grishin ${ }^{3}$, V.L. Solovianov ${ }^{3}$, \\ K. Jacobs ${ }^{4}$, and G.T. Zwart ${ }^{4}$ \\ 1 Spin Physics Center, University of Michigan, Ann Arbor, MI 48109-1120 \\ ${ }^{2}$ Indiana University Cyclotron Facility, Bloomington, IN 47408-0768 \\ 3 IHEP, Protvino, Russia 142 284 \\ ${ }^{4}$ MIT-Bates Linear Accelerator Center, 21 Manning Ave., Middleton, MA 01949
}

\begin{abstract}
.
We recently used a vertical-field rf-dipole magnet to study the spin-flipping of a $120 \mathrm{MeV}$ horizontally polarized proton beam stored in the presence of a nearly-full Siberian snake in the IUCF Cooler Ring. The spin was flipped by ramping the rfdipole's frequency through an rf-induced depolarizing resonance. After optimizing the frequency ramp parameters, we used multiple spin-flips to measure a maximum spinflip efficiency of $86.5 \pm 0.5 \%$ in April 2000, and $92.5 \pm 0.5 \%$ in June 2000. The spin-flip efficiency was apparently limited by the maximum achievable current in the rf-dipole. This result indicates that spin-flipping a stored polarized proton beam should be possible in high energy rings such as RHIC (and perhaps HERA in the future), where Siberian snakes are utilized and the dipole rf-flipper-magnets should be quite practical. During the June 2000 run, a new faster technique of locating the rf depolarizing resonance frequency was developed.
\end{abstract}

* Supported by research grants from the U.S. Department of Energy and the U.S. National Science Foundation.

** E-mail: alilin@umich.edu

(a) Also at: Moscow State University, Moscow, Russia.

(b) Also at: Portland Physics Institute, Portland, OR 97201, USA.

CP570, SPIN 2000, $14^{\text {th }}$ International Spin Physics Symposium, edited by K. Hatanaka et al. (C) 2001 American Institute of Physics 0-7354-0008-3/01/\$18.00 


\section{INTRODUCTION}

Siberian snake experiments in the IUCF Cooler Ring have been instrumental in understanding the spin dynamics of a polarized proton beam in a storage ring. In this past year, we studied spin-flipping of a polarized proton beam at the Cooler Ring. Frequent reversals of the beam polarization direction should help to significantly reduce systematic errors in a scattering experiment's spin asymmetry measurements. We previously used an rf solenoid to spin-flip, with $97 \pm 1 \%$ efficiency, a horizontally polarized proton beam stored in the Cooler Ring containing a Siberian snake. [1] However, a solenoid's spin rotation decreases linearly with energy because of the Lorentz contraction of its $\int B \cdot d l$; thus, a solenoid would be impractical for spin-flipping in high energy rings. On the other hand, a transverse rf dipole's spin rotation remains constant, independent of energy and thus should be more practical at high energies. As a first step, we earlier succeeded in spin-flipping efficiently with an rf-dipole in the absence of a Siberian snake. [2]

In a storage ring, each proton's spin precesses around the Stable Spin Direction (SSD) with a frequency of:

$$
f_{s}=f_{c} \nu_{s}
$$

where $f_{c}$ is the circulation frequency, and $\nu_{s}$ is the spin tune, which is the number of spin precessions during one turn around the ring. With a Siberian snake in the ring, the spin tune is:

$$
\nu_{s}=\frac{1}{\pi} \cos ^{-1}(\cos (\pi G \gamma) \cos (\pi s))
$$

where $G=(g-2) / 2=1.792847$ is the proton's anomalous magnetic moment, $\gamma$ is

its Lorentz energy factor, and $s$ is the snake strength. For a full $(100 \%)$ snake, $s=1$, and then $\nu_{s}=\frac{1}{2}$, independent of energy.

\section{Spin-flipping with a full Siberian snake}

One can spin-flip a horizontally polarized proton beam in a ring with a Siberian snake using a depolarizing resonance induced by a vertical-field rf-dipole; similarly, a horizontal-field rf-dipole can spin-flip a vertically polarized beam. A resonance occurs when the rf magnetic field's frequency is synchronized with the spin tune and the circulation frequency according to:

$$
f_{r}=f_{c}\left(k \pm \nu_{s}\right)
$$

where $\mathrm{k}$ is an integer. This resonance condition allows coherent kicks to build up and rotate the spin about the vertical axis by $180^{\circ}$ causing a spin-flip. 


\section{Spin-flipping parameters}

The rf resonance strength $\epsilon$ is given by:

$$
\epsilon=\frac{\theta_{s}}{\pi}=\frac{G e \int B \cdot d l}{2 \pi m_{p} v} \text { for an rf dipole, }
$$

where $\theta_{s}$ is the rf-dipole's spin rotation angle, $m_{p}$ is the proton's mass and $v$ is the proton's velocity. By varying the rf dipole frequency from below to above the resonance frequency $f_{r}$, one can cross the depolarizing resonance. After this crossing, the final beam polarization, $P_{f}$, is related to the initial beam polarization, $P_{i}$, by the Froissart-Stora form:

$$
P_{f}=P_{i}\left\{2 \exp \left[\frac{-\left(\pi \epsilon f_{c}\right)^{2}}{\Delta f / \Delta t}\right]-1\right\},
$$

where $\epsilon$ is the resonance strength, and $\Delta f / \Delta t$ is the resonance crossing rate for the frequency range $\Delta f$ during a ramp time $\Delta t$.

Three distinct conditions for the frequency variation rate $\Delta f / \Delta t$ are:

\begin{tabular}{lll}
\hline Rate & Polarizations & Effect \\
\hline Fast crossing: & $P_{f} \approx P_{i}$ & Little or no depolarization \\
Medium crossing: & $P_{i}>P_{f}>-P_{i}$ & Depolarization \\
Slow crossing (adiabatic): & $P_{f} \approx-P_{i}$ & Spin-flip \\
\hline
\end{tabular}

The spin-flip efficiency is defined as:

$$
\eta=\frac{-P_{f}}{P_{i}} .
$$

To maximize the spin-flip efficiency, one must carefully choose $\epsilon, \Delta f$, and $\Delta t$.

For a description of the apparatus used in this experiment, including the rfdipole, the IUCF Cooler Ring, the Siberian snake, and the polarimeter, the reader is referred to Ref. [3]. The $120 \mathrm{MeV}$ horizontally polarized proton beam in the Cooler Ring was obtained using the new Cooler Injector Polarized Ion Source (CIPIOS) and the new Cooler Injection Synchrotron. The beam polarization was about (50-60) $\pm 2 \%$ after the $7 \mathrm{MeV}$ Linac. At $120 \mathrm{MeV}$, the circulation frequency in the Cooler Ring was $f_{c}=1.59784 \mathrm{MHz}$.

With a nearly-full Siberian snake in the Ring, from Eq. (2), the spin tune $\nu_{s}$ is very near but not exactly equal to $\frac{1}{2}$. Therefore, Eq. (3) implies that two closely spaced rf depolarizing resonances, $f_{r}^{-}$and $f_{r}^{+}$, exist:

\begin{tabular}{lll}
\hline & April 2000 run & June 2000 run \\
\hline Center of 2 resonances & $1.5 f_{c}=2.39676 \mathrm{MHz}$ & $0.5 f_{c}=0.79892 \mathrm{MHz}$ \\
$f_{r}^{-}$ & $f_{c}\left(2-\nu_{s}\right)$ & $f_{c}\left(1-\nu_{s}\right)$ \\
$f_{r}^{+}$ & $f_{c}\left(1+\nu_{s}\right)$ & $f_{c}\left(0+\nu_{s}\right)$ \\
\hline
\end{tabular}

With a snake strength $s$ of $1.01, \nu_{s}$ was about 0.505 ; thus, the $f_{r}^{-}$resonance was:

slightly below $1.5 f_{c}$ for $\mathrm{k}=2$ for April 2000 run; and

slightly below $0.5 f_{c}$ for $\mathrm{k}=1$ for June 2000 run. 


\section{APRIL 2000 RESULTS}

In the April 2000 run, the $f_{r}^{-}$resonance was first located using the much stronger rf solenoid. Once found, this resonance was mapped out by the radial polarization response to varying the rf dipole's frequency, as shown in Fig 1.

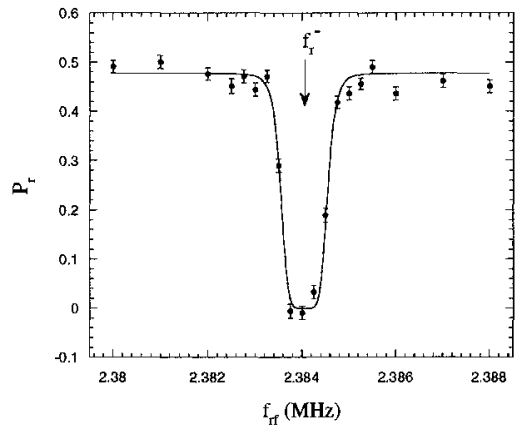

Fig. 1. The measured radial proton polarization plotted against the rf-dipole's frequency; arrow shows $f_{r}^{-}$. [3]

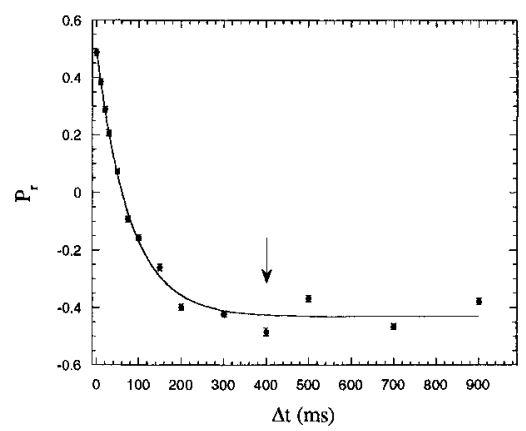

Fig. 2 Radial polarization plotted against the ramp time for a fixed frequency range of $\Delta f=10 \mathrm{kHz}$. [3]

The parameter $\Delta t$ was then optimized as shown in Fig. 2; it indicated that spin fips with slow rf ramp times.

In addition, remnant polarization of the beam was measured after various number of flips. From these graphs, a maximum spin-flip efficiency of $86.5 \pm 0.5 \%$ was obtained.

\section{JUNE 2000 RESULTS}

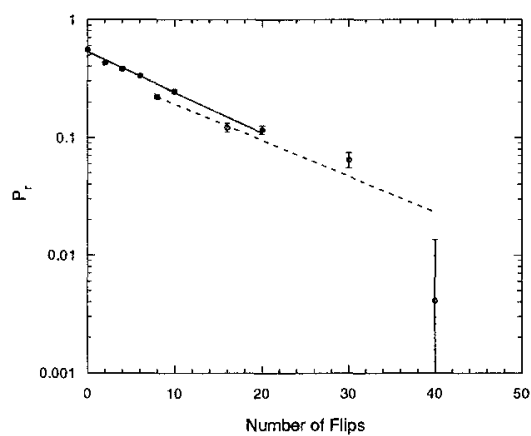

Fig. 3 Radial proton polarization plotted against number of spin-flips. The two fitted lines gave a spin-flip efficiency $\eta$ of about $92.5 \pm 0.5 \%$.

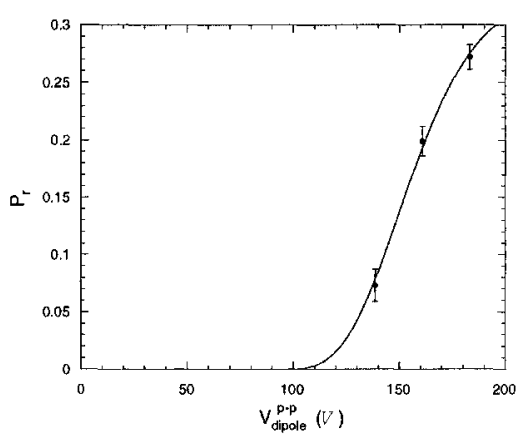

Fig. 4 Maximum radial polarization after ten spin-flips plotted against the peak-topeak dipole voltage. 
In June 2000, CE-69 enhanced the rf dipole strength by sweeping through a lower resonant frequency of $f_{r}^{-}=f_{c}\left(1-\nu_{s}\right)$. The data for multiple spin-flips is shown in Fig. 3; the two curves were fitted to $P_{n}=P_{i} \cdot \eta^{n}$, where $\eta$ is the efficiency and $n$ denotes the number of flips. The maximum spin-flip efficiency was found to be increased to $92.5 \pm 0.5 \%$.

Fig. 4 shows how the maximum spin-flip efficiency grows with the rf dipole's strength; a higher spin-flip efficiency should be possible with a higher strength.

During the June run, a new method was developed to locate the rf depolarizing resonance as outlined below:

- first make a large frequency sweep range, containing the spinflip;

- then divide the frequency sweep range into two equal sweeps;

- continue subdividing the range, always keeping the ones containing the spin-flip.

This method was much more efficient and has the advantage of observing the actual spin-flips, instead of a depolarizing dip.

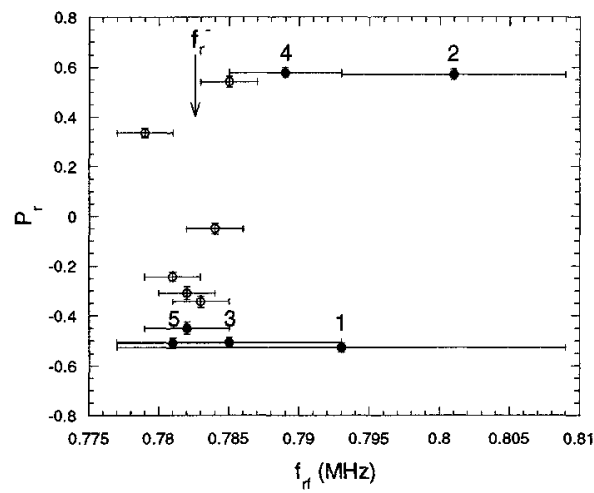

Fig. 5 Radial proton polarization plotted against the rf-dipole's central frequency, with widths indicating the frequency range; arrow shows $f_{r}^{-}$.

Such a process is shown graphically in Fig. 5 by points 1 to 5 , which were done sequentially. Notice also that, once roughly located, the resonance was mapped out by (small) equal frequency ranges of $\pm 2 \mathrm{kHz}$ around the resonance. The arrow indicates the resonance frequency $f_{r}^{-}$thus obtained; the same value was later obtained by mapping the polarization at many fixed frequency points around the resonance.

We thank the staff of IUCF for the successful operation of the Cooler Ring.

\section{REFERENCES}

1. B.B. Blinov et al., Phys. Rev. Lett. 81, 2906 (1998); V.A. Anferov et al., Proc. 13th Intl. Symposium on High Energy Spin Physics, eds. N.E. Tyurin et al., (World Scientific, Singapore, 1999) p. 503.

2. Spin flipping a stored polarized proton beam with an rf dipole, V.A. Anferov et al., Phys. Rev. ST-AB 3, 041001 (2000).

3. Spin-fipping with an rf-dipole and a full Siberian snake, B.B. Blinov et al., Phys. Rev. ST-AB 3, 104001 (2000). 